\title{
Knowledge, attitudes, and practices towards COVID-19 among residents of Gujarat, India
}

\author{
Dharmesh Domadia ${ }^{1}$, Jigisha Patadiya ${ }^{2}$, Prakash Bhabhor ${ }^{3 *}$
}

\author{
${ }^{1}$ AMI Park Society, Subhanpura, Baroda, Gujarat, India \\ ${ }^{2}$ Department of Pediatric, Government Medical College, Surat, Gujarat, India \\ ${ }^{3}$ Department of Pharmacology, GMERS Medical College, Gotri, Vadodara, Gujarat, India
}

Received: 12 June 2021

Revised: 13 July 2021

Accepted: 14 July 2021

*Correspondence:

Dr. Prakash Bhabhor,

Email: drpbhabhor@gmail.com

Copyright: $($ ) the author(s), publisher and licensee Medip Academy. This is an open-access article distributed under the terms of the Creative Commons Attribution Non-Commercial License, which permits unrestricted non-commercial use, distribution, and reproduction in any medium, provided the original work is properly cited.

\begin{abstract}
Background: India, having diverse demographic profile, any single measure to curtain COVID-19 spread will become ineffective by default. Hence, determining knowledge and behaviours of population regarding COVID-19 is must to extract and decide on an effective approach to reduce the spread of COVID-19. For the same, we conducted KAP study. Methods: It was a KAP study of adult residents of various districts of Gujarat, India; consisting questions with multiple responses pertaining to knowledge, attitude, and practices towards COVID-19. Responses were analysed and represented by their percentage. Knowledge related questions' responses were statistically analysed based upon responder's age, gender, education level.

Results: Knowledge scores of male and female participants were 7.03 and 6.96. Mean knowledge score of age group of 18-30 years, differed significantly to that of age group of >60 years. Study participants' scores didn't differ statistically according their education levels. 91.3\% participants felt that COVID-19 can be more effectively managed if person has been diagnosed or tested earlier in the disease. $69.8 \%$ participants felt that patients of COVID-19 infection can be safely treated at home. $74.4 \%$ of participant were in favour of lockdown if need arises. $77 \%$ participants were willing to take COVID-19 vaccine.

Conclusions: Knowledge level didn't differ for any gender or education level. Knowledge scores of populations above age of 60 years were statistically less as compared to the scores of participants aged between 18 to 30 years. TV news channels and newspapers were most sought way of getting information for current study population.
\end{abstract}

Keywords: COVID-19, Knowledge, Attitude, Practice, Gujarat

\section{INTRODUCTION}

Since infection of one 55 years old individual from Hubei province in China, COVID-19 became alarmingly spreading across the globe. ${ }^{1}$ Since, outbreak in December 2019, the novel coronavirus disease (COVID-19) has spread like a wild fire and spared no nation from its havoc. United States, India, Brazil and Russia are most affected ones, aggregating more than half of worldwide cases. Number of new COVID-19 cases per day reported in India suggests that India may become most affected country. Even following extensive measures like countrywide lockdowns, mass media awareness campaigns and extensive case searching and surveillance, new cases of COVID-19 are still on raise till date. With a population of more than 1.3 billion, India has ample potential to become worst affected country of the pandemic. Due to various demographic profiles of Indian population, any one single measure seems inefficient to control the upraise of pandemic in India. 
In order to derive and decide an efficient strategy to curtail the raising new COVID-19 cases, it becomes essential to find out current mental status and practices of population regarding COVID-19. As a part of the same, we planned a study to assess knowledge, attitude and practices towards COVID-19 of residents of Gujarat, India, a one of the worst affected states of country, India.

\section{METHODS}

We conducted a cross-sectional web-based knowledge attitude and practice analysis survey of adult residents of Gujarat, India, which was carried out between January 2021 to June 2021. It was conceptualized as an independent research, hence ethical approval from an independent institutional ethics committee was obtained. Study was executed online by utilising google forms after the ethical approval. It consisted ten, nine and seven questions with multiple responses pertaining to knowledge, attitude, and practices towards COVID-19, respectively. Snow ball sampling method was utilized for study participant selection. All the people who had access to mobile communication device with internet facility and willing to take part in the study were included and were analysed for the results. Total population of Gujarat in mid of year of 2020 was estimated to be $63,872,399 .^{2}$ With $90 \%$ confidence level, $5 \%$ margin of error, sample size of 273 is required for effective representation of the population size of 63872399 of Gujarat. We recorded 321 responses, and all were represented in the data for current study $(\mathrm{N}=321)$. Knowledge related questions' responses were analysed based upon responder's age, gender, education level and marital status by utilising one-way ANOVA. Microsoft excel (Ver -15.0.4433.1506) was used for data entry; and for analysis, Graphpad prism 9.0.2 (161) trial version was used.

\section{RESULTS}

Study received 321 responses and all were considered for analysis. Table 1 shows basic demographic characteristics of study population along with statistical analysis of knowledge scores of subgroups- gender wise, age wise, marital status wise and education wise. Knowledge scores of male and female participants were 7.03 and 6.96, respectively which didn't differ statistically. Groups were also subdivided according to age, and knowledge scores of various age groups like 18 to 30 years, 31 to 45 years, 46 to 60 years and $>60$ years were compared with each other with one-way ANOVA. Mean knowledge score of age group of 18-30 years- 7.09 differed significantly to mean knowledge score of age group of $>60$ years- 5.33 $(\mathrm{p}=0.0320) .91 .3$ percent participants felt that COVID-19 can be more effectively managed if person has been diagnosed or tested earlier in the disease. 69.8 percent participants felt that patients of COVID-19 infection can be safely treated at home. 81.3 percent participant opined that common public is not fully aware about COVID-19 infection and disease caused by it (Figure 1). 94.1 percent participants opined that COVID-19 transmission can be curtailed if people are equipped with information about the same. 74.4 percent of participant were in favour of lockdown if COVID-19 infection becomes rampant again, while $18 \%$ were against it (Figure 2).

Table 1: Basic demographic characteristics of study population along with their COVID-19 knowledge scores (out of total score 10) (scores are presented as mean $\pm \mathrm{SD})(\mathrm{N}=321)$.

\begin{tabular}{|llll|}
\hline Groups & Sub-groups & Number of responders (\%) & Knowledge scores \\
\hline Gender & Male & $150(46.73)$ & $6.96 \pm 1.247$ \\
\hline \multirow{2}{*}{ Age (years) } & Female & $171(53.27)$ & $7.03 \pm 1.008$ \\
& 18 to 30 & $251(78.19)$ & $7.09 \pm 1.038^{*}$ \\
& 31 to 45 & $56(17.45)$ & $6.77 \pm 1.308$ \\
& 46 to 60 & $11(3.43)$ & $6.27 \pm 1.421$ \\
\cline { 2 - 4 } & $>60$ & $3(0.93)$ & $5.33 \pm 1.528$ \\
\hline Marital status & Married & $67(20.87)$ & $6.69 \pm 1.258^{\wedge}$ \\
& Unmarried & $254(79.13)$ & $7.08 \pm 1.075$ \\
\hline Education & Primary & $0(0)$ & - \\
& Secondary & $23(7.17)$ & $6.96 \pm 1.022$ \\
& College & $243(75.70)$ & $6.96 \pm 1.115$ \\
& Professional degree & $55(17.13)$ & $7.16 \pm 1.214$ \\
\hline
\end{tabular}

Note: $* \mathrm{P}$ value $=0.0320$ when comparing knowledge scores of age group of 18-30 years to age group of $>60$ years, comparison carried out by one-way ANOVA; ${ }^{\wedge}=\mathrm{P}$ value $=0.0109$ when comparing knowledge scores of married population to unmarried population, comparison carried out unpaired $t$ test.

A total 83.2 percent of participant felt that children will become more vulnerable to the infection caused by COVID-19 if schools, colleges are reopened, and henceforward 59.2 percent of participants felt that government should not reopen schools or college till COVID-19 pandemic gets controlled (Figure 3).

In addition, $97.2 \%$ participants followed social distancing measures, $96.9 \%$ used hand sanitizer when going out of 
their homes and $99.1 \%$ wore face masks. 97.8 percent participants will consult a physician if they get infection of COVID-19 (Figure 4).

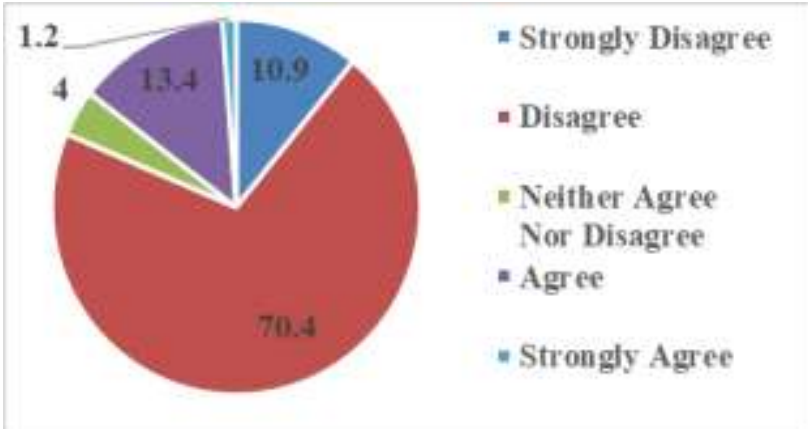

Figure 1: Responses of study participants to the question- "Do you think that general population is fully aware of COVID 19 and disease caused by it?" $(\mathrm{N}=321)$.

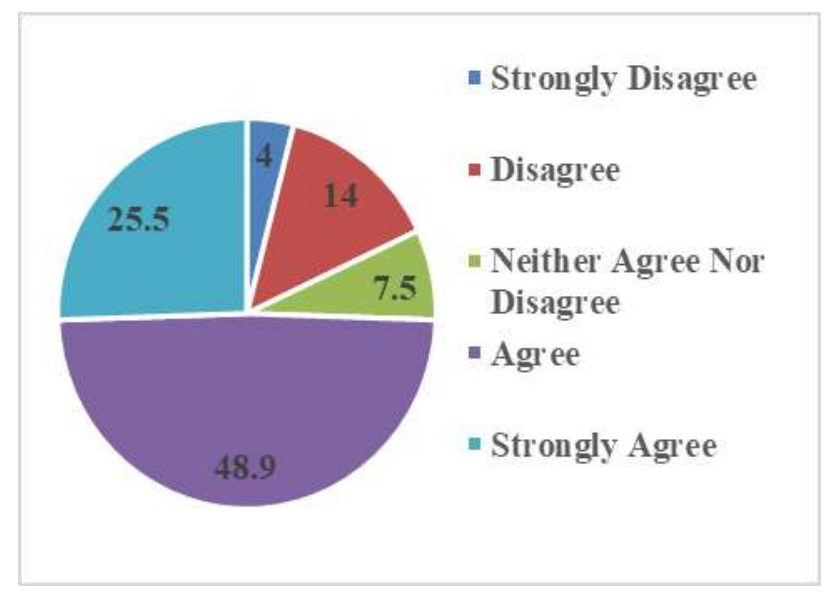

Figure 2: Responses of study participants to the question- "Do you think the government should announce a lockdown again if the COVID-19 infection is too high?" $(\mathrm{N}=321)$.

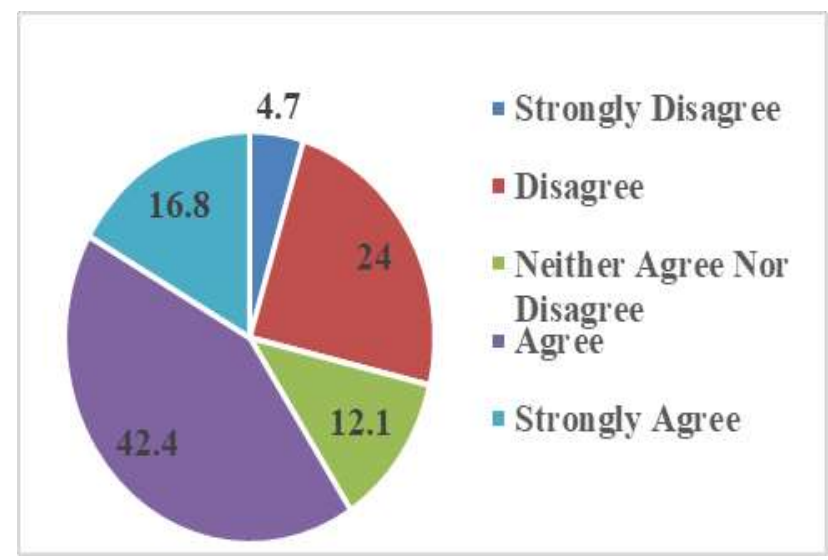

Figure 3: Responses of study participants to the question- "Do you think school-colleges should be closed for even longer to prevent children getting infected with COVID-19?" ( $=321)$.

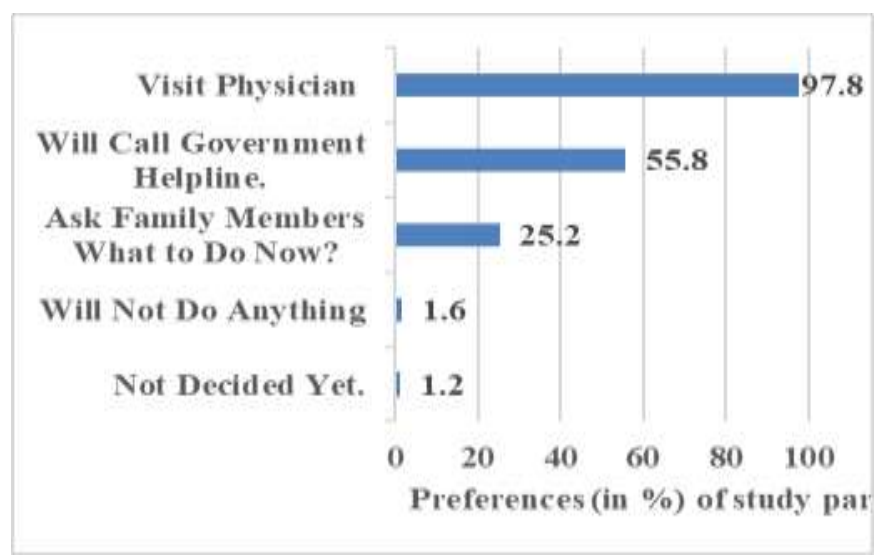

Figure 4: Multiple preferences (in \%) of study participants if they fall ill $(\mathrm{N}=321$, multiple responses were allowed).

A total 77 percent participants will take COVID-19 vaccine upon availability (Figure 5). 10.3 percent will not vaccinate their child for COVID-19 (Figure 6). TV news channels followed by newspaper were the most common source of information for $81.6 \%$ and $71.3 \%$ of participants, respectively (Figure 7).

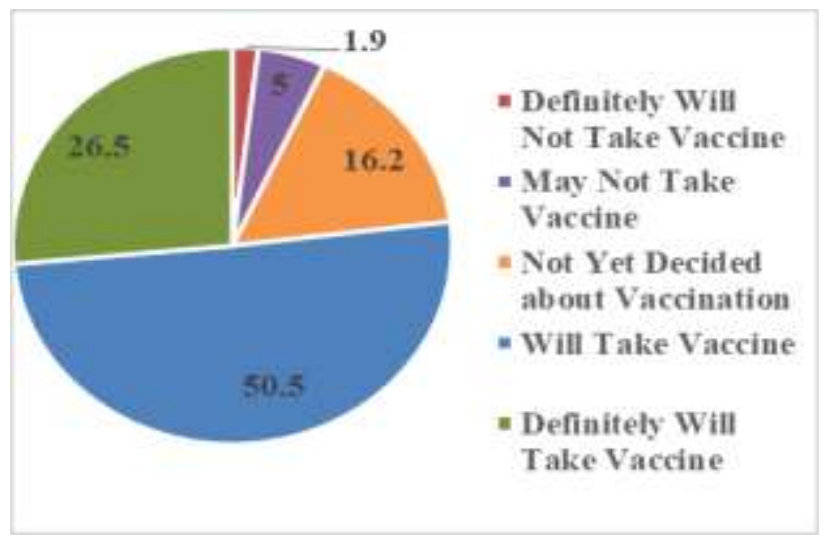

Figure 5: Preferences of study participants whether they will take vaccine against COVID-19 $(\mathrm{N}=321)$.

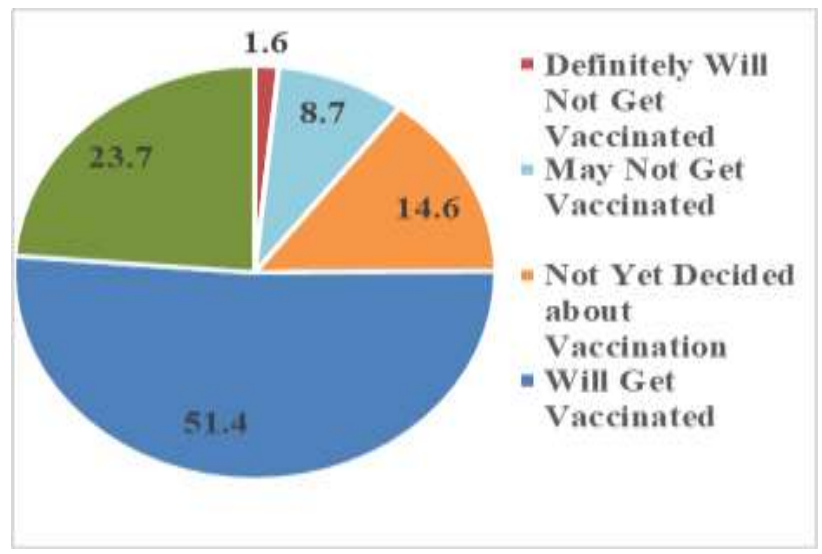

Figure 6: Preferences of study participants whether they will get their own or household children vaccinated against COVID-19 $(\mathrm{N}=321)$. 


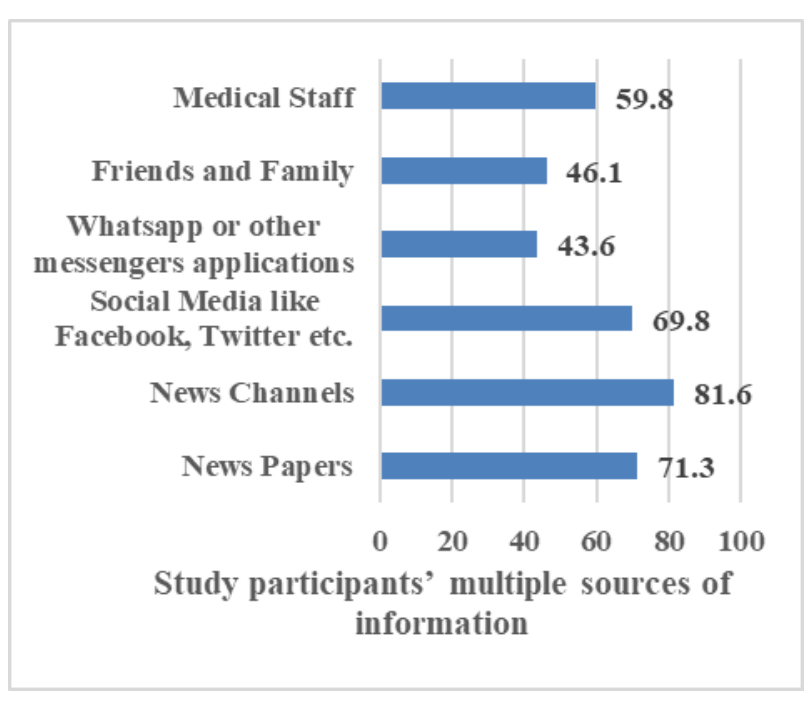

Figure 7: Study participants' multiple sources of information (in \%) for COVID-19 ( $N=321$, multiple responses were allowed).

\section{DISCUSSION}

The aim of the study was to assess knowledge, attitude and practices towards COVID-19 infection and its control measures. In the section of knowledge assessment for COVID-19, participants were asked about COVID-19 causative organism, disease symptoms, incubation period and diagnostic test. To these questions, participants reported, difficulty in breathing $(98.8 \%)$ followed by fever (98.4\%) to be the most common symptoms of COVID19 infection, which is in concert to a finding of another study carried out by Walid et al, who reported fever (97.6\%) to be most common symptom of COVID-19 as perceived by their study participants. ${ }^{3} 89.4$ percent of study participants correctly identified incubation period of COVID-19 infection. Table 1 also shows mean scores of participants according to their demographic categories. Knowledge scores were found to be adequate among various gender, educations and other age subgroups except subgroup of $>60$ years, where the mean score was 5.33. Here, age groups were subdivided and knowledge scores of this subgroups- 18 to 30 years, 31 to 45 years, 46 to 60 years and $>60$ years were compared with each other with oneway ANOVA. Mean knowledge score of age group of 1830 years- 7.09 differed significantly to mean knowledge score of age group of $>60$ years- $5.33(\mathrm{p}=0.0320)$. This finding is in line with a study done by Ferdous et al. who reported that younger people had significantly higher knowledge regarding COVID19 as compared to older age people. ${ }^{4}$ Similarly, groups were subdivided according to their marital status and their mean knowledge scores were compared with each other with unpaired t test. Mean knowledge score of married group- 6.69 differed significantly to mean knowledge score of unmarried population- $7.09 \quad(\mathrm{p}=0.0109)$. Reason behind this difference might be that, average age of unmarried participant was less as compared to the married participants. Study participants were also divided according to their education levels like primary, secondary, college, and professional degree qualification and their mean knowledge scores were also compared with each other which didn't differed significantly.

All 321 participants responded to the questions related to the attitude towards COVID-19 infection. 91.3 percent participants felt that COVID-19 can be more effectively managed if person has been diagnosed or tested earlier in the disease and even $20.9 \%$ participant felt that COVID19 is not at all a serious disease. 69.8 percent participants felt that patients of COVID-19 infection can be safely treated at home while 24.3 percent felt that it becomes difficult to manage COVID-19 infection if patient is treated at home. Strikingly, in spite of widespread government awareness campaigns, $12.1 \%$ participants yet believed that majority of patient infected with COVID-19 dies because of it. A study carried out by Srichan et al. reported that study participants perceived that their information regarding COVID-19 were insufficient. ${ }^{5}$ Similarly, current study also found that, 81.3 percent participant opined that common public is not fully aware about COVID-19 infection and disease caused by it (Figure 1), yet 94.1 percent participants were optimistic about that COVID-19 transmission can be curtailed if people are equipped with information about the same. During the first wave of COVID-19 pandemic, there was an outcry about lockdown and its economic effect on population, especially lower and lower middle class. But 74.4 percent of current study participants felt lockdown can be called for if COVID-19 infection becomes rampant again (Figure 2). 83.2 percent of participants feared about children becoming more vulnerable to the infection caused by COVID-19 if schools, colleges are reopened, and henceforward 59.2 percent of participants opined that government should not reopen schools or college till COVID-19 pandemic gets controlled (Figure 3).

Due to wide spread government campaigns and awareness tactics, majority participants followed COVID-19 aversion strategies like $97.2 \%$ followed social distancing measures, 96.9\% used hand sanitizer while being out of their homes and $99.1 \%$ wore face masks. This findings are in concert with findings of a previous study carried out by Pal et al. ${ }^{6}$ It was shocking that $1.6 \%$ participants will not visit doctor or call government helpline if they become infected with COVID-19, while majority $97.8 \%$ will prefer to consult a physician if they get infection of COVID-19 (Figure 4). In contrast to a study carried out in Greece, where vaccine uptake willingness percentage was found to be $43 \%$, current study reported that $77 \%$ participants were willing to take COVID-19 vaccine upon availability, while $6.9 \%$ had decided to skip vaccination (Figure 5). ${ }^{7} 10.3$ percent of participants were not willing to get their child vaccinated for COVID-19 (Figure 6), reason behind this aversion might be that vaccine safety and efficacy among paediatric population is still being evaluated by many vaccine providers and their data is not publically available for their reference. 
TV news channels followed by newspaper were the most common source of information for $81.6 \%$ and $71.3 \%$ of participants, respectively (Figure 7). Similarly, a previous study carried out by Srichan et al. reported television as major source of information regarding COVID-19. ${ }^{5}$ But, $16.6 \%$ participants were sceptical about the authenticity of their source of information, while only 27.5 percent participants had full trust on their source of information.

\section{Limitations}

This was a web-based survey, which required access to mobile communication device with internet facility. Hence, data of people who had not access to these facilities was not obtained in this study. In addition, because of this, study may have excluded the participation by lower economic class with less resources.

\section{CONCLUSION}

Current study explored knowledge of population of Gujarat, India regarding COVID-19 infection and control measures. May be because of various government awareness campaigns targeted towards COVID-19, knowledge scores of participants be it male or female, or any education levels were adequate and comparably similar. Knowledge scores of populations above age of 60 years were statistically less as compared to the scores of participants aged between 18 to 30 years. TV news channels and newspapers were most sought way of getting information for current study population over other information dispersal modalities.

\section{Recommendations}

Current study data shows that awareness measures were not adequate for the population above age of 60 years as their knowledge scores were statistically less as compared to the scores of participants aged between 18 to 30 years. This group of elderly people, being most vulnerable to development of COVID-19 infection related complications, current study observations stresses improvement required in information dispersal targeted towards elderly population. As, TV news channels and newspapers being most sought way of getting information for current study population, these modalities should be prioritized over other information dispersal modalities, in order to increase awareness regarding current pandemic situation

\section{ACKNOWLEDGEMENTS}

Authors of current study are grateful to Dr. Vishalkumar K. Vadgama, Assistant Professor, Department of
Pharmacology, Government Medical College, Bhavnagar, Gujarat for providing assistance in creating study questioners and survey tools.

Funding: No funding sources

Conflict of interest: None declared

Ethical approval: The study was approved by the Institutional Ethics Committee

\section{REFERENCES}

1. South China Morning Post. China's first confirmed Covid-19 case traced back to November 17, 2020. https://www.scmp.com/news/china/society/article/30 74991/coronavirus-chinas-first-confirmed-covid-19case-traced-back. Accessed on 16 March 2021.

2. Gujarat population, 2021. Available at: http://www.populationu.com/in/gujarat-population. Accessed on 16 March 2021.

3. Qerem WA, Jarab AS. COVID-19 Vaccination Acceptance and Its Associated Factors Among a Middle Eastern Population. Front Public Health. 2021;9:632914.

4. Ferdous MZ, Islam MS, Sikder MT, Mosaddek ASM, Valdivia JA, Gozal D. Knowledge, attitude, and practice regarding COVID-19 outbreak in Bangladesh: An online-based cross-sectional study. PLoS One. 2020;15(10):239254.

5. Srichan P, Apidechkul T, Tamornpark R, Yeemard F, Khunthason S, Kitchanapaiboon S, et al. Knowledge, attitudes and preparedness to respond to COVID-19 among the border population of northern Thailand in the early period of the pandemic: a cross-sectional study. WHO South East Asia J Public Health. 2020;9(2):118-5.

6. Pal R, Yadav U, Grover S, Saboo B, Verma A, Bhadada SK. Knowledge, attitudes and practices towards COVID-19 among young adults with Type 1 Diabetes Mellitus amid the nationwide lockdown in India: A cross-sectional survey. Diabetes Res Clin Pract. 2020;166:108344.

7. Papagiannis D, Malli F, Raptis DG, Papathanasiou IV, Fradelos EC, Daniil Z, et al. Assessment of Knowledge, Attitudes, and Practices towards New Coronavirus (SARS-CoV-2) of Health Care Professionals in Greece before the Outbreak Period. Int J Environ Res Public Health. 2020;17(14):4925.

Cite this article as: Domadia D, Patadiya J, Bhabhor P. Knowledge, attitudes, and practices towards COVID-19 among residents of Gujarat, India. Int J Basic Clin Pharmacol 2021;10:992-6. 